\title{
Ein Modell zur Bilanzierung des holzerntebeding- ten Nährstoffentzugs auf Schweizer Waldböden
}

\author{
Renato Lemm \\ Oliver Thees \\ Urs Hensler \\ Jörg Hässig \\ Anton Bürgi \\ Stephan Zimmermann
}

\author{
Eidgenössische Forschungsanstalt für Wald, Schnee und Landschaft $(\mathrm{CH})^{*}$ \\ Eidgenössische Forschungsanstalt für Wald, Schnee und Landschaft $(\mathrm{CH})$ \\ Vermessungsamt der Stadt Winterthur, Fachstelle Geoinformation $(\mathrm{CH})$ \\ GEO Partner AG $(\mathrm{CH})$ \\ Eidgenössische Forschungsanstalt für Wald, Schnee und Landschaft $(\mathrm{CH})$ \\ Eidgenössische Forschungsanstalt für Wald, Schnee und Landschaft $(\mathrm{CH})$
}

\section{A model for balancing nutrient extraction caused by timber harvesting on Swiss forest soils}

\begin{abstract}
A model named NBM was developed to judge quantitatively the risks of soil nutrient extraction due to timber harvesting in Switzerland. The model can be used for forest enterprises as well as for larger regions. It assesses nutrient extraction at the level of the single stand as part of a scenario analysis. The extraction of nine nutrients as a result of harvesting is quantified under conditions with different silvicultural and bucking methods. The results are then compared to the soil potentials and changes in these potentials. The simulation results are assessed with respect to sustainability. Two criteria are used to evaluate the long-term risk of nutrient deficiency. The validity of the model in a practical context is limited as more soil informations would be needed. The paper describes structure and functions of the model, its computational implementation, and the results obtained from its first application in the forest enterprise of Bremgarten, Wohlen, Waltenschwil. It ends with a critical analysis of this first implementation of the model.
\end{abstract}

Keywords: nutrients deficiency, balance of nutrients, timber harvesting, modeling doi: $10.3188 /$ szf.2010.0401

*Zürcherstrasse 111, CH-8903 Birmensdorf, E-Mail renato.lemm@wsl.ch
$\mathrm{D}$ ie intensive Energieholznutzung und die Rationalisierung der Holzernte am Steilhang bedingen eine zunehmende Nutzung von Vollbäumen. Gerade für die Bewirtschaftung des Schutzwaldes kann dieses Verfahren eine wirtschaftlich sehr effiziente Methode darstellen. Aktuell wird es in der Schweiz auf $12 \%$ der Waldfläche angewendet, in den Alpen und Voralpen ist es noch etwas häufiger (Brändli 2010). Durch die Entnahme von Ästen, Reisig und Nadeln/Blättern erhöht sich jedoch der Nährstoffentzug gegenüber der reinen Schaftholznutzung beträchtlich. Hierdurch kann es je nach Ausgangssituation des Waldstandortes und Intensität der Nutzung zu Beeinträchtigungen der Nährstoffversorgung kommen. Für eine nachhaltige Waldbewirtschaftung ist es daher erforderlich, die Risiken des holzerntebedingten Nährstoffentzugs zu kennen. Vor diesem Hintergrund werden in der Schweiz durch die Eidgenössische Forschungsanstalt für Wald, Schnee und Landschaft (WSL) und auch im benachbarten Ausland (z.B. Seifert et al 2006) Instrumente zur Bilanzierung des Nährstoffhaushalts entwickelt, welche modellbasiert eine situationsspezifische Szenarioanalyse auf quantitativer Grundlage erlauben. Besonderheit des Schweizer Ansatzes ist es, dass der erntebedingte Nährstoffentzug nicht nur quantifiziert, sondern auch in Beziehung zum Nährstoffpotenzial im Boden gesetzt wird. Der folgende Beitrag zeigt den Aufbau und die Funktionsweise des an der WSL für Schweizer Wälder entwickelten Nährstoffbilanzierungsmodells, skizziert die IT-Umsetzung, visualisiert die Ergebnisse der ersten Anwendung im WSL-Forschungswald Bremgarten, Wohlen, Waltenschwil und mündet in eine kritische Analyse der bisherigen Erfahrungen.

\section{Das Nährstoffbilanzierungsmodell}

\section{Zweck und Anforderungen}

Um die Nährstoffentzüge durch die Holzernte bei unterschiedlicher Nutzungsintensität zu bewerten, wurde ein flexibles Nährstoffbilanzierungsmodell (NBM) entwickelt. Das Modell sollte: 


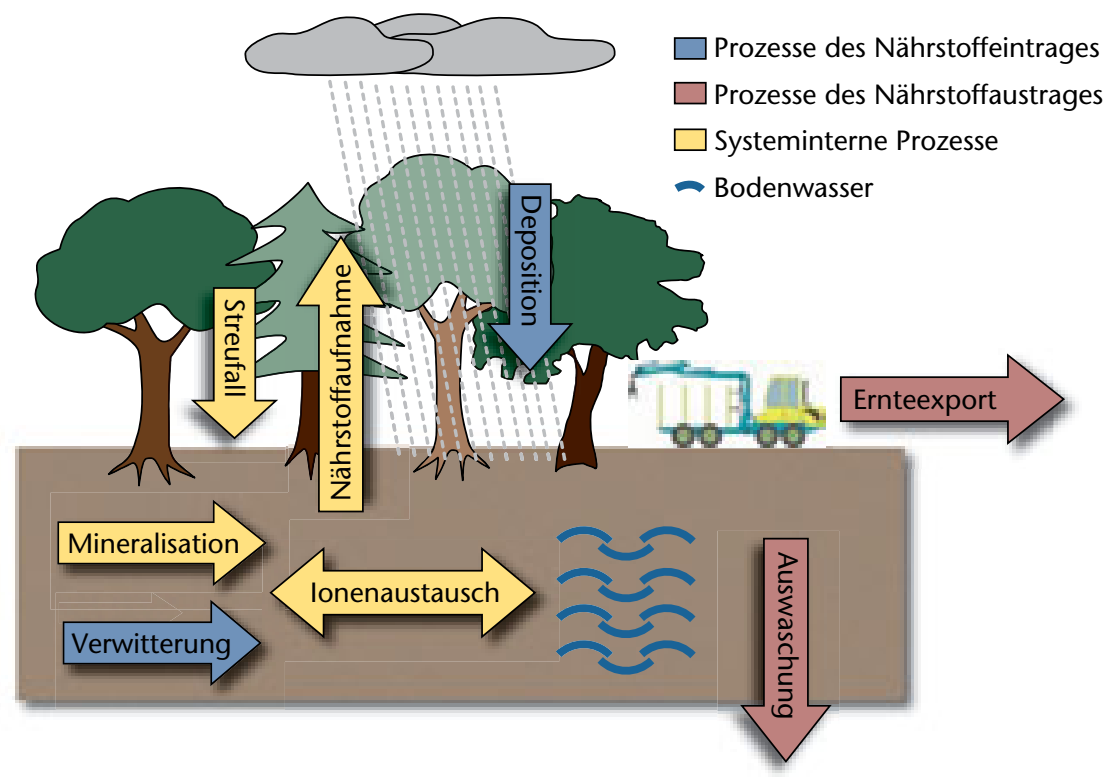

Abb 1 Nährstoffflüsse im Nährstoffbilanzierungsmodell (NBM). Die systeminternen Prozesse spielen sich innerhalb des Bilanzrahmens ab und werden im Modell nicht abgebildet.

- den Einfluss der Holzernte auf die Nährstoffversorgung für konkrete Waldstandorte quantifizieren und bewerten,

- dabei die üblicherweise auftretenden und mehr oder weniger gut quantifizierbaren Ein- und Austräge sowie den Nährstoffvorrat im Boden berücksichtigen,

- bei unterschiedlichen Waldbauvarianten und Beständen (auch Mischbeständen) anwendbar sein, - die Analyse unterschiedlicher Aushaltungsvarianten (Derbholz- bis Vollbaumnutzung) erlauben, - die Makronährelemente Stickstoff (N), Phosphor (P), Kalium (K), Calcium (Ca), Magnesium (Mg) und Schwefel (S) sowie die Mikronährelemente Eisen (Fe) und Mangan (Mn) und im Weiteren das nützliche Nährelement Natrium ( $\mathrm{Na}$ ) einbeziehen, ${ }^{1}$ - die Nährstoffentzüge detailliert nach Baumkompartimenten erfassen,

- lange Bewirtschaftungszeiträume bis zu einer Umtriebszeit abdecken,

- die Berechnungen und Bewertungen durch eine Softwarekomponente ausführen und mithilfe eines GIS visualisieren.
Bestandes- und Standortgrössen Anteil Laubwald Bonität Laubwald Bonität Nadelwald Forstregion Waldgesellschaft
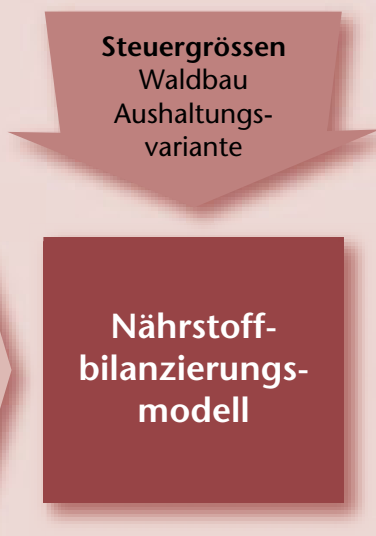

\section{Aufbau und Funktionsweise}

Das NBM berücksichtigt die Komponenten Nährstoffvorrat im Boden, Lufteintrag (Deposition), Verwitterung des Ausgangsgesteins, Auswaschung und Ernteexport (Abbildung 1). Die systeminternen Prozesse sowie der Eintrag durch nicht geerntete Biomasse werden nicht abgebildet, da sie den Bilanzrahmen nicht verlassen. Das NBM erfordert als Input eine vereinfachte pflanzensoziologische und bestandesspezifische Charakterisierung des Standorts. Dazu wird einerseits dem jeweiligen Bestand diejenige Waldgesellschaft gemäss pflanzensoziologischer Karte mit dem grössten Flächenanteil zugeordnet. Andererseits werden die Bestände anhand der Bestandesbeschreibung in Laubholz (repräsentiert durch Buche) und in Nadelholz (repräsentiert durch Fichte) differenziert. Die Laub- und Nadelwaldbonität wird aus der Waldgesellschaft abgeleitet und der Deckungsgrad der Bestockung der Bestandesbeschreibung entnommen. Über die beiden Steuergrössen Waldbauvariante und Aushaltungsvariante lassen sich verschiedene Bewirtschaftungsszenarien abbilden. Als Resultat wird die Standortgefährdung durch den Nährstoffentzug bei der Holzernte ausgegeben und visualisiert (Abbildung 2).

Den Aufbau und den Informationsfluss des NBM zeigt Abbildung 3. Die Berechnung der Derbholz-Biomasse pro Durchmesserklasse auf Brusthöhe (BHD) während einer Umtriebszeit erfolgt mit dem dynamischen Forstbetriebssimulationsmodell (FBSM; Erni \& Lemm 1995). Als Eingangsgrössen dienen Baumart, Bonität und Waldbaukonzept. Mittels Kompartimentsanteilen und Ernteverlusten werden die entnommenen Mengen an Biomasse je Kompartiment und BHD-Klasse berechnet. Schlussendlich wird anhand der Aushaltungsvarianten und der Nährstoffgehalte je Kompartiment der holzerntebedingte Nährstoffexport ermittelt.

Die Standortgefährdung wird in zwei Schritten erfasst. Im ersten werden für jeden Nährstoff die beiden Nachhaltigkeitsfaktoren Nachlieferung pro Nährstoff (NNN) und Gesamtvorrat pro Nährstoff (NGV) berechnet. Der NNN stellt den Lufteintrag, die Verwitterung und die Auswaschung dem Ernteexport gegenüber. Damit lässt sich feststellen, ab welcher Nutzungsintensität der Ernteexport nicht mehr durch die Einträge gedeckt ist. Der NGV setzt den Nährstoffvorrat ins Verhältnis zu Lufteintrag, Verwitterung, Auswaschung und Ernteexport während einer Umtriebszeit. Auf diese Weise kann bestimmt werden, nach wie vielen Umtriebszeiten (hypothetisch) der Vorrat aufgebraucht sein wird (Göttlein et al 2007). Im zweiten Schritt werden dann die berechneten Nachhaltigkeitsfaktoren der einzelnen

\footnotetext{
1 Kupfer $(\mathrm{Cu})$ und Zink (Zn) konnten nicht berücksichtigt werden, da es zu diesen beiden Elementen nicht genügend Grundlagen gibt.
}

Ausgabegrössen Bewertung der Standortgefährdung durch Holzernte 
Abb 3 Aufbau und Informationsfluss des Nährstoffbilanzierungsmodells (NBM).

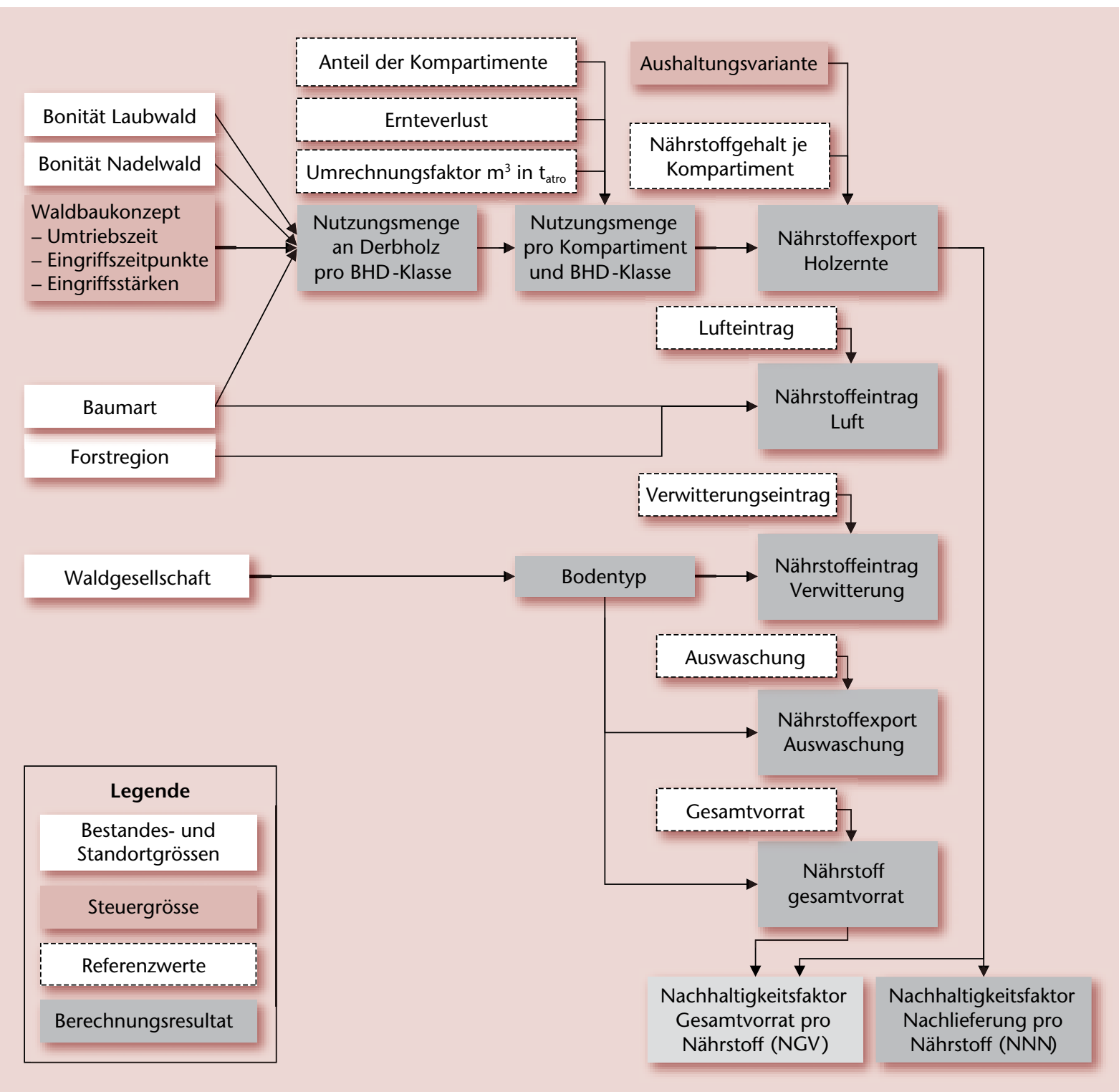

Nährstoffe aufgrund ihrer Bedeutung für das Pflanzenwachstum gewichtet und die Gefährdung des Standorts als Ganzes beurteilt.

\section{Schritt 1: Berechnung der Nachhaltigkeits- faktoren}

Der NNN ist abhängig vom Lufteintrag, vom Verwitterungseintrag, vom Auswaschungsexport sowie vom Ernteexport; der NGV wird zusätzlich vom Gesamtvorrat des Nährstoffes bestimmt (Hässig et al 2009). Die Berechnung von NNN und NGV erfolgt gemäss Formeln 1 und 2:

$$
\begin{aligned}
& N N N_{r f}=\frac{V E_{r}+D E_{f}-L E_{r}}{N E} \\
& N G V_{w r f}=\frac{G V_{w}}{L E_{r}+N E-D E_{f}-V E_{r}}
\end{aligned}
$$

wobei

NNN ${ }_{\text {rf }}$ Nachhaltigkeitsfaktor Nachlieferung pro Nährstoff NGV wrf Nachhaltigkeitsfaktor Gesamtvorrat pro Nährstoff

$G V_{w} \quad$ Gesamtvorrat je Waldgesellschaft in $\mathrm{kg} / \mathrm{ha}$

$V E_{r} \quad$ Eintrag durch Verwitterung je Verwitterungsklasse pro Umtriebszeit in $\mathrm{kg} /(\mathrm{ha} \times \mathrm{U})$
$D E_{f} \quad$ Nährstoffeintrag über die Luft je Forstregion pro Umtriebszeit (Deposition) in $\mathrm{kg} /(\mathrm{ha} \times \mathrm{U})$

$L E_{r} \quad$ Nährstoffauswaschung je Verwitterungsklasse pro Umtriebszeit (Leaching) in $\mathrm{kg} /(\mathrm{ha} \times \mathrm{U})$

NE Nährstoffexport pro Umtriebszeit (Ernteexport) in $\mathrm{kg} /(\mathrm{ha} \times \mathrm{U})$

$w=1 . .5$ Waldgesellschaften nach Ellenberg \& Klötzli (1972) mit Nährstoffvorräten (Walthert et al 2004, Blaser et al 2005, Zimmermann et al 2006)

$r=1 . .5$ Verwitterungsklassen in Abhängigkeit der Bodentypen (Hässig et al 2009)

$f=1 . .5$ Forstregionen der Schweiz: Jura, Mittelland, Voralpen, Alpen, Alpensüdseite

Eintrag durch Verwitterung des Ausgangsgesteins Die Verwitterung ist die Freisetzung von Nährstoffen aus der chemischen Auflösung von Muttergestein. Je nach Ausgangsgestein, Höhenlage und klimatischen Verhältnissen variieren die Verwitterungsraten. Für alle Nährstoffe ausser N sind Verwitterungsraten pro Verwitterungsklasse vorhanden (Hässig et al 2009).

Mit dem regionalen Steady-state-Bodenchemiemodell «Profile» (Sverdrup 1990) konnten (basie- 
rend auf der Berechnung von Massenbilanzen für die Säureneutralitätskapazität) für Podsol Verwitterungsraten der basischen Kationen $\mathrm{K}, \mathrm{Ca}, \mathrm{Mg}$ und $\mathrm{Na}$ ermittelt und unter der Berücksichtigung von durchwurzelbarer Bodentiefe und Höhenstufe für die Schweiz abgeleitet werden. Über die Verwitterungsraten für Podsol als Basis und die Zuordnung vieler in der Schweiz vorkommenden Bodentypen in Klassen aufgrund ihrer Säureneutralisationskapazität nach Rihm (1994) konnten den Waldgesellschaften die Verwitterungsraten zugeordnet werden. Entsprechend der Klasse wurden die Verwitterungsraten für Podsol vervielfacht und konnten so für andere Bodentypen berechnet werden. Für die Nährstoffe Mn, Fe, P und S musste auf Mazzarino (1981) sowie auf Sigg \& Stumm (1994) zurückgegriffen werden.

\section{Lufteintrag}

Der Lufteintrag ist regional sehr unterschiedlich, wobei er bei einzelnen Nährstoffen bedeutsam für die Nährstoffversorgung ist (BFS 2002). Daher wird der Lufteintrag, sofern die Datengrundlage es erlaubt, spezifisch für die Forstregion sowie für Nadel-, Laub- und Mischwald berücksichtigt. Der Eintrag von $\mathrm{K}, \mathrm{Ca}, \mathrm{Mg}$, Na und $\mathrm{S}$ wurde aus den Messungen auf den Flächen der langfristigen Waldökosystem-Forschung (LWF) der WSL hergeleitet (Thimonier et al 2005). Für den N-Eintrag wurde das Stickstoffdepositionsmodell des Bundesamtes für Umwelt verwendet (Bafu 2007). Für Mn, Fe und $P$ wurden Daten aus dem nahe gelegenen Schwarzwald beigezogen (Heyn 1989).

\section{Auswaschung}

Ein wichtiger Austrag ist die Auswaschung (Leaching) von Nährstoffen in Bodenschichten tiefer als $60 \mathrm{~cm}$ oder ins Grundwasser. Sie hängt von diversen Kriterien wie den spezifischen Eigenschaften des Bodens ab und kann sehr hoch sein. Für K, Ca, Mg und S wurden Messwerte der LWF herangezogen (Thimonier et al 2005). Die Auswaschung ist abhängig vom Bodentyp, welcher über die Waldgesellschaft dem betrachteten Standort zugeordnet wird. Bei der Berechnung der N-Auswaschung werden die Deposition, die Immobilisation, die Denitrifikation sowie der Ernteexport berücksichtigt (Hässig et al 2009).

\section{Nährstoffvorrat}

Der Vorrat an Nährstoffen im Boden ist wichtig für die langfristige Nährstoffversorgung der Pflanzen. Die Vorratswerte wurden Walthert et al (2004), Blaser et al (2005) und Zimmermann et al (2006) entnommen. Die horizontspezifischen Vorräte wurden bis auf $60 \mathrm{~cm}$ Bodentiefe aufsummiert. Für die mittel- und flachgründigen Böden mit 40 beziehungsweise $20 \mathrm{~cm}$ durchwurzelbarer Tiefe erfolgte eine anteilsmässige Reduktion der Vorräte. Der Gesamtvorrat ist abhängig vom Bodentyp, welcher wiederum über die Waldgesellschaften dem betrachteten Standort zugeordnet wird.

\section{Ernteexport}

Der Ernteexport entspricht dem Nährstoffentzug durch die Holzernte. Dabei werden sämtliche Nutzungen über eine Umtriebszeit berücksichtigt, d.h. alle Durchforstungen und die Endnutzung. Der oberirdische Teil eines Baumes besteht aus den Kompartimenten Schaftholz, Äste und Reisig sowie Blätter und Nadeln, die mit unterschiedlichen Nährstoffgehalten ausgestattet sind. Stärkere Buchen können erhebliche Mengen Astderbholz (Durchmesser $>7 \mathrm{~cm}$ mit Rinde) aufweisen. Mit der Nutzungsmenge nach Kompartimenten pro BHD-Klasse, dem Nährstoffgehalt in den Kompartimenten und den Kompartimentsanteilen pro Baum (bezogen auf das Schaftholz) lässt sich der Nährstoffexport für Fichten- und Buchenvollbäume berechnen (Formel 3).

$$
N E=\sum_{i=1}^{7} \sum_{k \in A V}\left(N M_{k i} \times N G_{k i}\right) \times\left(100 \%-E V_{k i}\right): 100 \%
$$

wobei

NE Ernteexport (Nährstoffexport durch Holzernte) in $\mathrm{kg} /(\mathrm{ha} \times \mathrm{U})$

$N M_{k i} \quad$ Nutzungsmenge pro Kompartiment (k) und BHDKlasse (i) in $t_{\text {atro }} /(h a \times U)$

Umrechnungsfaktor von $\mathrm{m}^{3}$ in $t_{\text {atro: }}$ Fichte: 0.379 , Buche; 0.558 (Schmidt 2003)

$N G_{k i} \quad$ Nährstoffgehalt pro Kompartiment und BHD-Klasse in $\mathrm{kg} / \mathrm{t}_{\text {atro }}$

$k=1 \quad$ Derbholz in Rinde

$k=2 \quad$ Astderbholz (nur Buche)

$k=3 \quad$ Äste und Reisig

$k=4 \quad$ Nadeln/Blätter

$i=1 . .710-\mathrm{cm}$-Intervalle (alle Bäume mit $B H D \geq 60 \mathrm{~cm}$ in $i=7$ )

$E V_{K I} \quad$ Ernteverlust bei Vollbaumnutzung pro Kompartiment und BHD-Klasse in \%

Je nach Aushaltungsvariante (AV) wird über unterschiedliche k summiert:

Derbholz $k=1$; Vollbaum Nadelholz $k=1,3$, 4; Vollbaum (Winter) Laubholz $k=1$, 2, 3; Vollbaum (Sommer) Laubholz $k=1,2,3,4$

Für die Berechnung der Nutzungsmenge an Derbholz pro BHD-Klasse während einer Umtriebszeit wurde das dynamische Forstbetriebssimulationsmodell (FBSM; Erni \& Lemm 1995) verwendet und in das NBM integriert. Der Modellkern, das Bestandesentwicklungsmodell, ist ein distanzunabhängiges Einzelbaummodell, wobei der Einzelbaum jeweils eine Durchmesserklasse repräsentiert. Als Eingangsgrössen dienen Bonitäten, Baumart, Deckungsgrad und Waldbaukonzept. Für drei Waldbaukonzepte (vgl. Kapitel «Steuergrössen») wird jeweils die Bestandesentwicklung simuliert. Dabei legt das Waldbaukonzept die Umtriebszeit sowie die Eingriffszeitpunkte und -stärken fest.

Für die Simulation der Aushaltungsvarianten ist es notwendig, die Nutzungsmengen aller Kompartimente zu kennen. Neben dem Derbholz werden 
bei der Buche die Kompartimente Astderbholz, Äste (Nichtderbholz) und Blätter und bei der Fichte die Kompartimente Reisig und Nadeln berücksichtigt. Die Nutzungsmengen der einzelnen Kompartimente werden durch die Multiplikation der Nutzungsmenge des Derbholzes pro BHD-Klasse mit einem dem Kompartiment entsprechenden Faktor berechnet (Brändli et al 2001, Rademacher \& Müller-Using 2004). Mit der Wahl der Aushaltungsvariante wird festgelegt, welche Kompartimente vom Standort entfernt werden. Die gewählte Aushaltungsvariante gilt für alle Eingriffe eines Umtriebes. Mit dem Ernteverlust kann zudem der prozentuale Anteil eines Kompartimentes bestimmt werden, der im Wald zurückbleibt.

Bei jeder Holzernte - auch bei der Vollbaumnutzung - bleibt immer ein Teil der Biomasse aller Kompartimente im Bestand zurück, was als Ernteverlust bezeichnet wird. Dieser Verlust wurde für die beiden Baumarten und für jedes Kompartiment separat definiert; er bewegt sich im Bereich von 10 bis $40 \%$ der berechneten Nutzungsmenge pro Kompartiment und wird von dieser abgezogen (Wittkopf 2005).

Die ermittelten Nutzungsmengen werden durch die Umtriebszeit dividiert; man erhält die durchschnittliche jährliche Nutzungsmenge pro Kompartiment und BHD-Klasse (in $\mathrm{m}^{3}$ ). Aus der Nutzungsmenge wird durch Multiplikation mit dem baumartenabhängigen Umrechnungsfaktor nach Schmidt (2003) die Trockenmasse berechnet. Der Export eines Nährstoffes ergibt sich aus der Multiplikation der Trockenmasse mit dem Nährstoffgehalt. Der Nährstoffgehalt ist pro Baumart, BHD-Klasse und Kompartiment definiert (Rademacher \& Müller-Using 2004). Der absolute Nährstoffexport wird

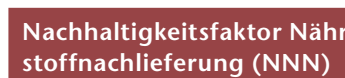

$1.1 \leq \mathrm{NNN}$

$0.9<\mathrm{NNN}<1.1$

$0.9<$ NNN $<1.1$

$\mathrm{NNN} \leq 0.9$

$\mathrm{NNN} \leq 0.9$

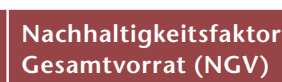

Gesamtvorrat (NGV)

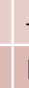

$\mathrm{NGV} \geq 10$

$\mathrm{NGV}<10$

$\mathrm{NGV} \geq 10$

$\mathrm{NGV}<10$

\begin{tabular}{|l|}
\hline Bewertung \\
\hline unproblematisch \\
unproblematisch \\
\hline indifferent \\
\hline unproblematisch \\
\hline problematisch \\
\hline
\end{tabular}

Tab 1 Bewertung eines Nährstoffes in Bezug auf die Nachhaltigkeitsfaktoren Nährstoffnachlieferung (NNN) und Gesamtvorrat (NGV).

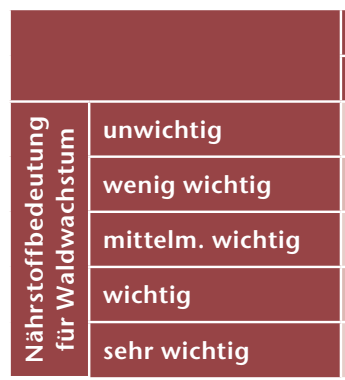

\begin{tabular}{|l|l|l|}
\hline \multicolumn{3}{|c|}{ ungewichtete Bewertung eines Nährstoffes } \\
\hline problematisch & indifferent & unproblematisch \\
\hline indifferent & unproblematisch & unproblematisch \\
\hline problematisch & indifferent & unproblematisch \\
\hline problematisch & indifferent & unproblematisch \\
\hline problematisch & problematisch & unproblematisch \\
\hline problematisch & problematisch & indifferent \\
\hline
\end{tabular}

Tab 2 Gewichtete Bewertung eines Nährstoffes in Bezug auf die Nachhaltigkeitsfaktoren Nährstoffnachlieferung (NNN) und Gesamtvorrat (NGV) unter Einbezug der Bedeutung eines Nährstoffes für das Waldwachstum. durch die Addition der Nährstoffexporte derjenigen Kompartimente ermittelt, welche bei der gewählten Aushaltungsvariante genutzt werden.

Die in Abbildung 3 gestrichelt eingezeichneten Referenzwerte des Nährstoffbilanzierungsmodells sind vorläufig nur für die beiden Baumarten Fichte und Buche definiert. Um die Simulation von Mischbeständen näherungsweise durchzuführen, werden entsprechend den Mischungsanteilen von Laub- und Nadelholz Reinbestände berechnet und zusammengesetzt. Stellvertretend für Laubbäume werden die Parameter für Buche und für Nadelbäume diejenigen für Fichte verwendet. Die Fichten und Buchen sind bezüglich ihrer Kompartimentsanteile Schaftderbholz, Rinde, Astderbholz, Reisig und Nadeln/Blätter sowie der Nährstoffgehalte in den Kompartimenten am besten untersucht.

Schritt 2: Bewertung der Standortgefährdung Die Bewertung eines Nährstoffes bezüglich NNN erfolgt mithilfe der drei Klassen «unproblematisch», «indifferent» und «problematisch» (Tabelle 1). Nährstoffe mit einem $\mathrm{NNN} \geq 1.1$ werden als unproblematisch deklariert. Resultate im Bereich des Wertes 1 sind schwierig zu beurteilen. Deshalb wird bei einem NNN zwischen 0.9 und 1.1 zusätzlich mit dem Nachhaltigkeitsfaktor NGV berechnet, wie lange es dauert, bis der Nährstoffgesamtvorrat aufgebraucht ist. Ist der $N G V \geq 10$, dauert es bei gleich bleibenden Bedingungen mehr als 10 Umtriebszeiten, bis der Gesamtvorrat aufgebraucht ist. Die Nachhaltigkeit wird in diesem Fall als unproblematisch beurteilt; bei einem NGV $<10$ wird sie hingegen als indifferent betrachtet. Bei einem $\mathrm{NNN} \leq 0.9$ wird ebenfalls zusätzlich der NGV berechnet, mit dem Unterschied, dass bei einem NGV $<10$ die Nachhaltigkeit des betreffenden Nährstoffes als problematisch angesehen wird.

Durch den Einbezug der Bedeutung eines Nährstoffes für das Waldwachstum wird die Bewertung weiter verfeinert (Tabelle 2 ). So kann zum Beispiel ein in der ungewichteten Skala als problematisch eingestufter Nährstoff aufgrund seiner geringen Bedeutung für das Waldwachstum in der gewichteten Bewertung als indifferent bewertet werden. Für die gewichtete Bewertung gelten die Nährstoffe $\mathrm{K}, \mathrm{Mg}$, P und S als wichtig, $\mathrm{Ca}, \mathrm{Mn}$ und $\mathrm{N}$ als mittelmässig wichtig, Fe als wenig wichtig und $\mathrm{Na}$ als unwichtig. ${ }^{2}$

Die Gesamtbewertung eines Standorts wird anhand der Anzahl von unproblematischen, indifferenten und problematischen Nährstoffen gemäss Tabelle 3 vorgenommen. Wenn alle Nährstoffe als unproblematisch klassiert sind, so wird auch der Standort als ungefährdet betrachtet. Bei einem bis

\footnotetext{
2 Bedeutung der Nährstoffe für die Forstgewächse: http://bfw. ac.at/inst3/publ/kilian/duenger/kap3.html (18.06.2010)
} 


\begin{tabular}{|c|c|c|c|}
\hline \multicolumn{3}{|c|}{ Anzahl Nährstoffbewertungen } & \multirow[t]{2}{*}{ Gesamtbewertung } \\
\hline unproblematisch & indifferent & problematisch & \\
\hline 9 & 0 & 0 & ungefährdet \\
\hline- & $\geq 1$ & 0 & indifferent \\
\hline- & - & 1 oder 2 & leicht gefährdet \\
\hline- & - & $\geq 3$ & gefährdet \\
\hline
\end{tabular}

Tab 3 Gesamtbewertung eines Standorts aufgrund der Nährstoffbewertungen.

zwei problematischen Nährstoffen gilt der Standort gutachtlich als leicht gefährdet. Sind mehr als zwei Nährstoffe problematisch, wird der Standort als gefährdet betrachtet.

\section{Bestandes- und Standortgrössen}

Die Bestandes- und Standortinformationen werden durch folgende Grössen erfasst:

- Anteil Laubwald: die Angabe des prozentualen Anteils des Laubwaldes an einem Standort. Dies erlaubt eine näherungsweise Bewertung der Standortgefährdung auch für Mischwälder.

- Bonität Laubwald und Bonität Nadelwald: Die Bonität als Mass für die Standortgüte des Laub- und des Nadelwaldes wird für die Simulation des Wachstums der Laub- und Nadelbäume verwendet.

- Forstregion: die Lage des Standortes in einer der fünf Schweizer Produktionsregionen Jura, Mittelland, Voralpen, Alpen und Alpensüdseite. Diese Angabe wird für die Bestimmung des Nährstoffeintrages durch die Luft benötigt.

- Waldgesellschaft: Die Waldgesellschaft nach Ellenberg \& Klötzli (1972) beschreibt die potenziell natürliche Vegetation, welche sich ohne menschlichen Einfluss am Standort einstellen würde. Mangels vorhandener Bodenkarten werden der Waldgesellschaft (als Eingangsgrösse) Bodentypen und Verwitterungsklassen zugeordnet (Hässig et al 2009).

\section{Steuergrössen}

Über das Waldbaukonzept und die Aushaltungsvariante kann das Modell gesteuert werden. Das Waldbaukonzept definiert die Eingriffzeitpunkte und -stärken in einem Bestand, um ein waldbauliches Ziel zu erreichen. In der vorliegenden Modellversion sind drei Waldbaukonzepteimplementiert (Rosset et al 2009):

- Wertholz (Wh): Primär wird Wertholz erzeugt, andere Sortimente nur, soweit sie als Kuppelprodukte anfallen. Die Z-Bäume werden durch drei Eingriffe stark begünstigt, es erfolgen kaum Eingriffe im Füllbestand. Durch starkes Begünstigen der Wertträger ist die durchschnittliche Umtriebszeit (Nadelholz 85-140 Jahre; Laubholz 100-150 Jahre) kürzer als beim Wertholz mit Energieholz als Kuppelprodukt, weil der Wertzuwachs früher kulminiert.

- Wertholz mit Energieholz als Kuppelprodukt (WEK): Primär wird Wertholz, zudem aber auch möglichst viel Biomasse produziert. Die drei Eingriffe er- folgen zugunsten der Z-Bäume, die stark begünstigt werden, aber auch im Füllbestand (Ernte von Energieholz). Die Umtriebszeit (Nadelholz 90-150 Jahre; Laubholz 120-150 Jahre) ist länger als beim reinen Energieholz, weil die Verjüngung erst bei der Kulmination des Wertzuwachses erfolgt.

- $\quad$ Energieholz (Eh): Es wird primär, aber nicht ausschliesslich Energieholz erzeugt. Die zwei bis drei Eingriffe erfolgen zugunsten von Z-Bäumen und im gesamten Füllbestand. Die Umtriebszeit ist kurz (Nadelholz 80-110 Jahre; Laubholz 80-100 Jahre), und die Verjüngung erfolgt ungefähr bei der Kulmination des Massenzuwachses. Die Nutzungsmenge ist kleiner als beim Waldbaukonzept Wertholz.

Die Aushaltungsvariante definiert, welche Kompartimente eines Baumes bei der Holzernte genutzt und somit aus dem Nährstoffkreislauf entfernt werden. Es kann aus drei Varianten ausgewählt werden:

- Vollbaum Sommer (VBS): Der Baum wird während der Vegetationsperiode geerntet. Sowohl Laubwie auch Nadelbäume werden vollständig (inkl. Blättern und Nadeln) aus dem Wald entfernt.

- Vollbaum Winter (VBW): Der Baum wird ausserhalb der Vegetationsperiode geerntet. Bei Laubbäumen bleiben die Blätter im Wald zurück, Nadelbäume werden vollständig genutzt.

- Derbholz (Dh): Es wird nur das Derbholz, d.h. das oberirdische Holzvolumen mit einem Mindestdurchmesser von $7 \mathrm{~cm}$ in Rinde, genutzt. Die Blätter und Äste respektive die Nadeln und das Reisig bleiben im Wald.

\section{Ausgabegrössen}

Um die Standortgefährdung durch den Nährstoffentzug bei der Holzernte zu berechnen und $\mathrm{zu}$ visualisieren, werden folgende Ausgabegrössen ermittelt:

- Berechnung und Bewertung von NNN und NGV für jeden Nährstoff.

- Gesamtbewertung der Gefährdung eines Standorts gestützt auf die Bewertung der einzelnen Nährstoffe bezüglich NNN und NGV sowie ihrer Bedeutung für das Waldwachstum.

Diese Bewertungen werden mithilfe eines geografischen Informationssystems (GIS) räumlich dargestellt.

\section{Realisierung des Nährstoff- bilanzierungsmodells als Software- komponente}

Die heutigen technischen Möglichkeiten bieten unterschiedliche Optionen, um ein Modell in einem einfach anzuwendenden Computerprogramm umzusetzen. Eine zweckmässige Möglichkeit bietet die von Lemm et al (2002) vorgeschlagene 
Modellierung und Entwicklung von komponentenbasierter Software. Solche Softwarebausteine gewährleisten einen flexiblen und vielfältigen Einsatz. Diese Methode hat sich auch bereits in unterschiedlichen Projekten bewährt (Lemm et al 2000, Erni \& Frutig 2004, Lemm \& Erni 2009). Für die Umsetzung des NBM wurde deshalb dieses Konzept verwendet.

Die realisierte Software liegt zurzeit als Prototyp vor. Es sind zwei Produkte implementiert:

\section{- .NET-Komponente, \\ - ArcGIS-Tool.}

\section{.NET-Komponente}

Die .NET-Komponente ist eine Softwarekomponente, welche auf Basis der .NET-Technologie von Microsoft implementiert wurde. In ihr ist die Kernfunktionalität des Modells umgesetzt. Sie erlaubt es, durch die Verarbeitung der Eingabe- und Steuergrössen eine Standortbewertung durchzuführen. Diese Komponente lässt sich in beliebige Computerprogramme, die auf der .NET- oder COM-Technologie basieren (z.B. Microsoft Office), integrieren.

Die in Abbildung 3 dargestellten Referenzwerte (z.B. «Nährstoffgehalt je Kompartiment» oder «Nährstoffeintrag Verwitterung») des NBM sind in Tabellen im csv-Format gespeichert. Dies sind Textdateien, welche sich zur Speicherung einfach strukturierter Daten wie zum Beispiel Tabellen eignen und bei Bedarf mit einem einfachen Textverarbeitungsprogramm angepasst werden können.

\section{ArcGIS-Tool}

Das ArcGIS-Tool ist eine Erweiterung für das geografische Informationssystem ArcGIS der Firma ESRI. Es dient als grafische Benutzerschnittstelle für die.NET-Komponente und ermöglicht eine einfache Steuerung bei der Verarbeitung von Geodaten. Mit dieser Software kann die Standortgefährdung über ein grösseres Waldgebiet (z.B. eine Gemeinde) berechnet, bewertet und mit dem Programm «ArcMap» als Karte visualisiert werden. Dies bietet die Möglichkeit, gefährdete Standorte zu lokalisieren und die waldbauliche Planung darauf auszurichten.

\section{Fallbeispiel Forstbetrieb Bremgarten, Wohlen und Waltenschwil}

Der Einfluss der Holzernte auf die Nährstoffbilanz wurde exemplarisch für den Wald des Forstbetriebes Bremgarten, Wohlen, Waltenschwil (BWW) untersucht. Dieser im Schweizer Mittelland gelegene Forstbetrieb bewirtschaftet eine Fläche von 676 ha und verfügt über einen Laubwaldanteil von 58\% (Abbildung 4). Als natürliche Waldgesellschaften nach Ellenberg \& Klötzli (1972; Abbildung 5) liegen in erster Linie Waldmeister-Buchen-Wälder (6a, 7a, 7aS, 7d, 7f, 7g) vor, in geringem Masse aber auch Orchideen-Buchen-Wälder (17) sowie Ahorn-, Erlen- und Eschenwälder (26f, 26g, 27g, 29, 29a).

Die Bewirtschaftung des Waldes wird durch den Forstbetrieb BWW sichergestellt. Traditionellerweise lag der Schwerpunkt auf der Produktion von Stammholz. Durch den Bau eines Nahwärmeverbundes in Bremgarten besteht neuerdings auch ein grosser Bedarf an Energieholz. Da der Forstbetrieb als primärer Lieferant für diese Holzschnitzelheizung auftritt, wurde die Nutzug in den letzten Jahren intensiviert. Unter diesen Voraussetzungen ist es von Interesse, wie sich die zusätzliche Nutzung auf die Nährstoffbilanz im Untersuchungsgebiet auswirkt.

Für die Durchführung der Fallstudie im Forstbetrieb BWW konnte auf bestehendes Datenmaterial zurückgegriffen werden. Laut Waldgesetz des Kan-

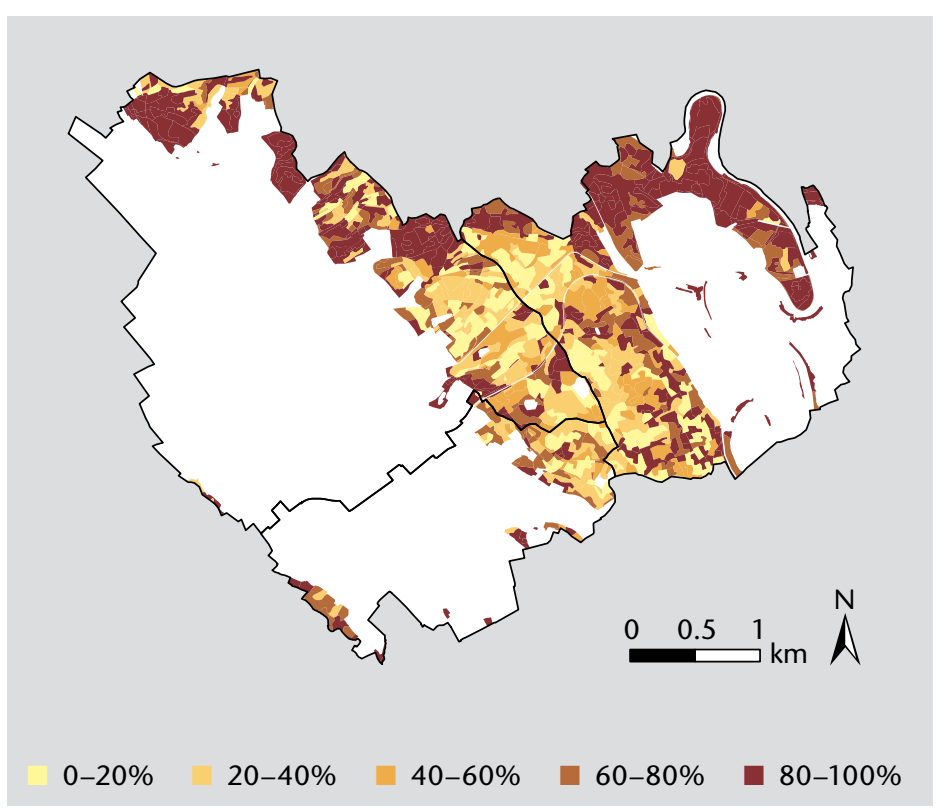

Abb 4 Laubwaldanteil auf Territorium der drei politischen Gemeinden Bremgarten, Wohlen und Waltenschwil.

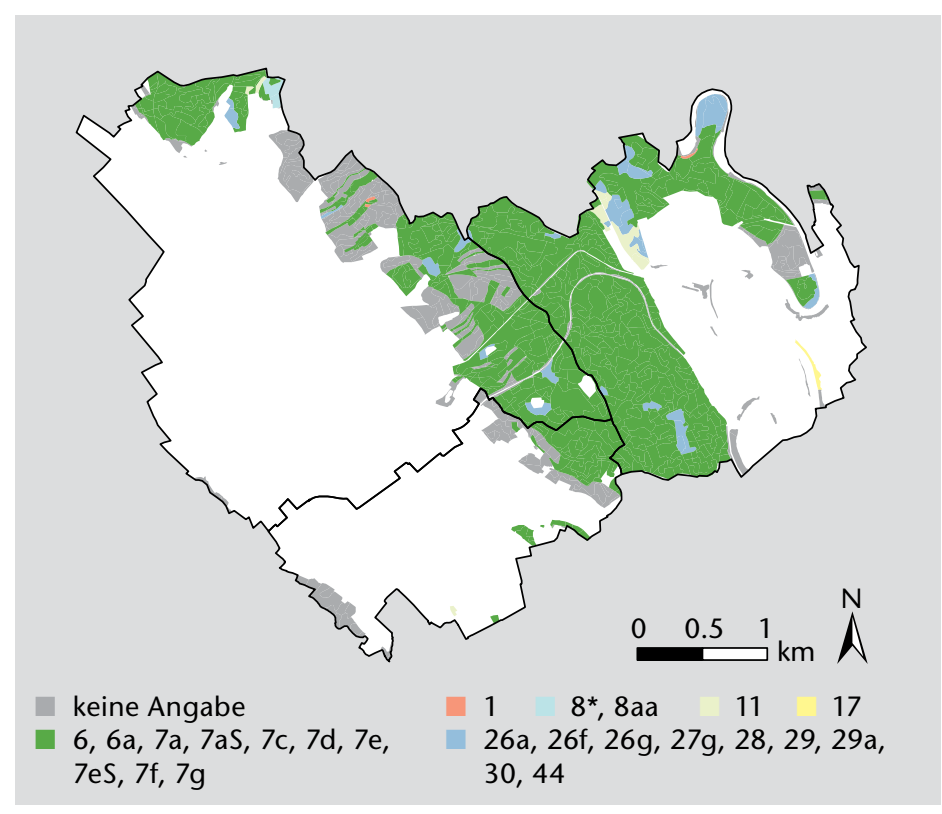

Abb 5 Waldgesellschaften im Forstbetrieb Bremgarten, Wohlen, Waltenschwil (BWW). 
tons Aargau vom 1. Juli 1997 sind die Forstbetriebe verpflichtet, Betriebspläne zu erstellen, welche Auskunft über die vorgesehene Bewirtschaftung der Wälder geben. Zentraler Teil dieser Betriebspläne ist die Bestandeskarte nach Vorgaben der Abteilung Wald des Kantons Aargau. Der Kanton stellt den Revierförstern dazu die Web-Applikation «BKOnline» zu Verfügung, welche seit einigen Jahren auch vom Forstbetrieb BWW eingesetzt wird. Diese Software erlaubt es einem Forstbetrieb, für die einzelnen Bestände die zentralen Informationen für die Bewirtschaftung digital zu erfassen und nachzuführen. Diese umfassen unter anderem die Ausdehnung, den prozentualen Anteil der Baumarten, die Bonitäten des Laub- und Nadelwaldes sowie die vorherrschende Waldgesellschaft.

\section{Nutzungsmenge}

Durch die Vollbaumnutzung kann die Biomassennutzung gesteigert werden (Abbildung 6). Im konkreten Fallbeispiel um 28\%. Bei der Variante Wertholz, Derbholz beträgt die durchschnittliche jährliche Nutzungsmenge an Biomasse $11.8 \mathrm{~m}^{3} /$ ha. Für den gesamten Forstbetrieb ergibt sich eine absolute jährliche Nutzungsmenge von $7771 \mathrm{~m}^{3}$. Demgegenüber beläuft sich bei der Variante Wertholz, Vollbaum Winter die durchschnittliche jährliche Nutzungsmenge auf $15.1 \mathrm{~m}^{3} /$ ha oder $9971 \mathrm{~m}^{3}$.

\section{Bewertung der Standortgefährdung}

In der Abbildung 7 sind die Gesamtbewertungen von vier Simulationen dargestellt, jeweils zwei Kombinationen der Waldbauvarianten «Wertholz» (Wh) und «Energieholz» (Eh) sowie der beiden Aushaltungsvarianten «Derbholz» (Dh) und «Vollbaum Winter» (VBW). 24\% des Waldes wurden in den Resultaten der Kategorie «keine Angabe» zugeordnet.
Dies hat zwei Gründe. Erstens befindet sich ein grosser Teil dieser Bestände im Eigentum von Privaten und wird nicht vom Forstbetrieb BWW bewirtschaftet. Wichtige Eingangsdaten waren daher nicht verfügbar. Zweitens wurde ein weiterer, kleiner Teil der Bestände aufgrund von Unstimmigkeiten in den Eingangsdaten der Kategorie «keine Angabe» zugeordnet.

Der Vergleich zwischen den beiden Waldbauvarianten Wh und Eh zeigt nur geringfügige Unterschiede. Es ist eine leicht stärkere Gefährdung beim Einsatz der Waldbauvariante Wh gegenüber Eh feststellbar, so zum Beispiel mehr «leicht gefährdete» bei der Derbholznutzung und mehr «gefährdete» bei der Vollbaumnutzung. Die leicht stärkere Gefährdung bei der Waldbauvariante Wh ist auf den höheren Biomasseentzug pro Jahr infolge intensiverer Bewirtschaftung zurückzuführen.

Augenfälliger ist der Unterschied bei den Aushaltungsvarianten. Die Gefährdung bei der Vollbaumnutung (VBW) nimmt gegenüber einer Derbholznutzung (Dh) deutlich zu. So beträgt bei der Variante Wh, Dh der Flächenanteil leicht gefährdeter Bestände $16 \%$, und man findet keine gefährdeten Bestände. Demgegenüber nimmt bei der Variante Wh,VBW der Flächenanteil leicht gefährdeter Standorte von $16 \%$ auf $62 \%$ zu, und 5\% der Standorte werden sogar als gefährdet eingestuft. Diese gefährdeten Standorte findet man insbesondere bei einem hohen Laubwaldanteil ( $\geq 90 \%)$, da in den Kompartimenten von Buchen die Nährstoffgehalte $(\mathrm{K}, \mathrm{Mg}$, S, P) zum Teil beträchtlich höher sind als bei Fichte.

\section{Bewertung bezüglich ausgewählter Nährstoffe}

Im Forstbetrieb ist bei Vollbaumnutzung im Winter und bei der Waldbauvariante Wertholz das Calcium auf weiten Teilen des Untersuchungsgebie-

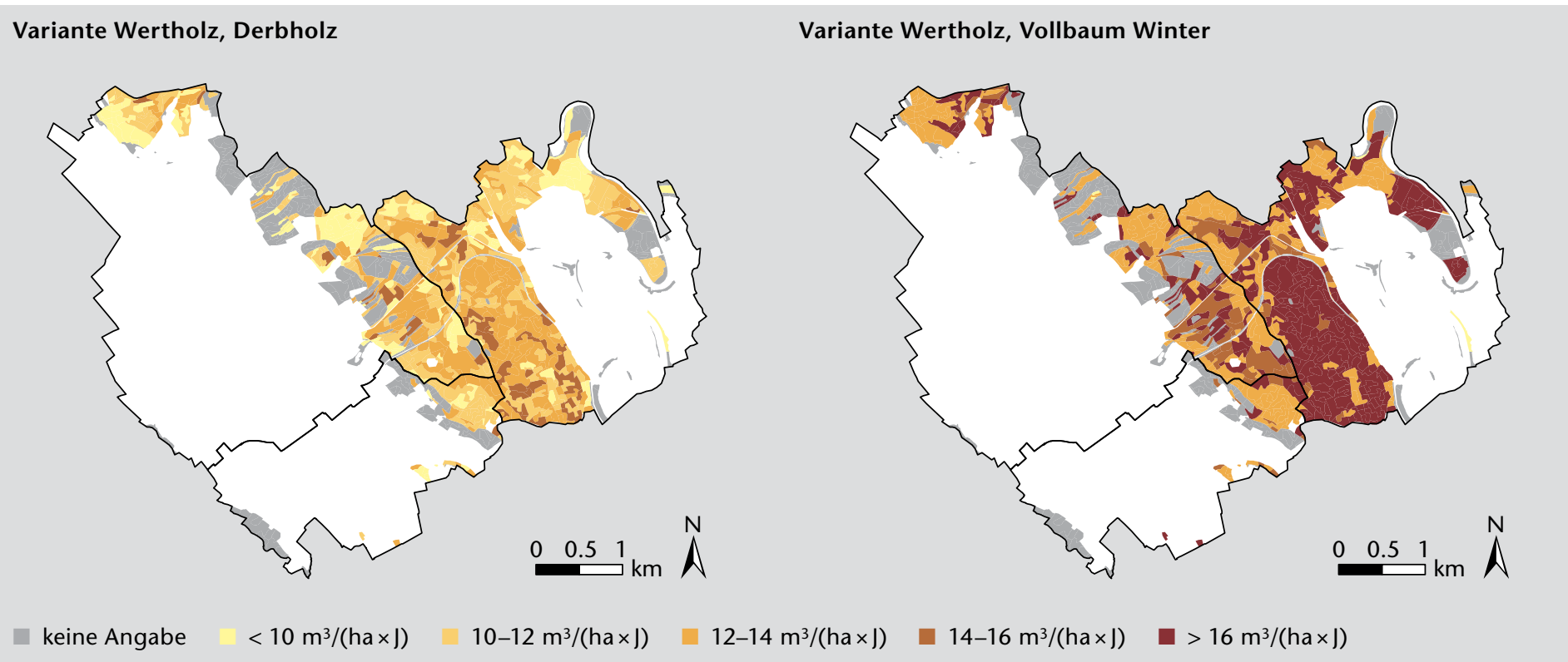

Abb 6 Jährliche Nutzungsmengen im Forstbetrieb Bremgarten, Wohlen, Waltenschwil (BWW) bei zwei verschiedenen Aushaltungsvarianten. Die grauen Flächen (keine Angabe) betreffen hauptsächlich den Privatwald auf Territorium der politischen Gemeinden Bremgarten, Wohlen und Waltenschwil. 


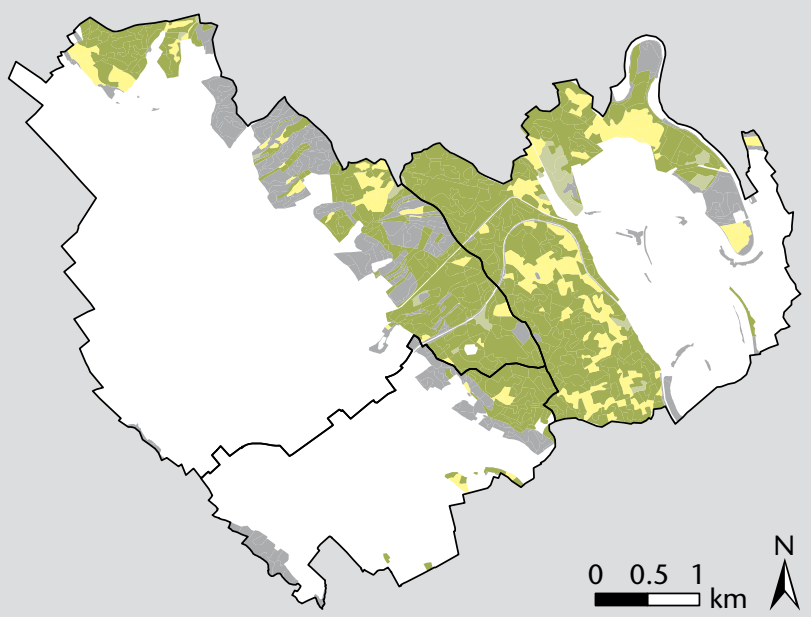

Variante Wertholz, Vollbaum Winter

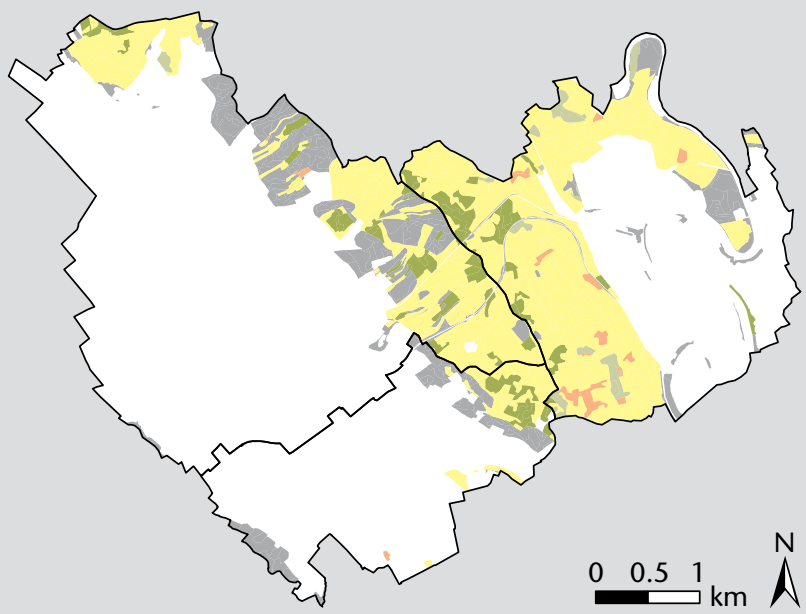

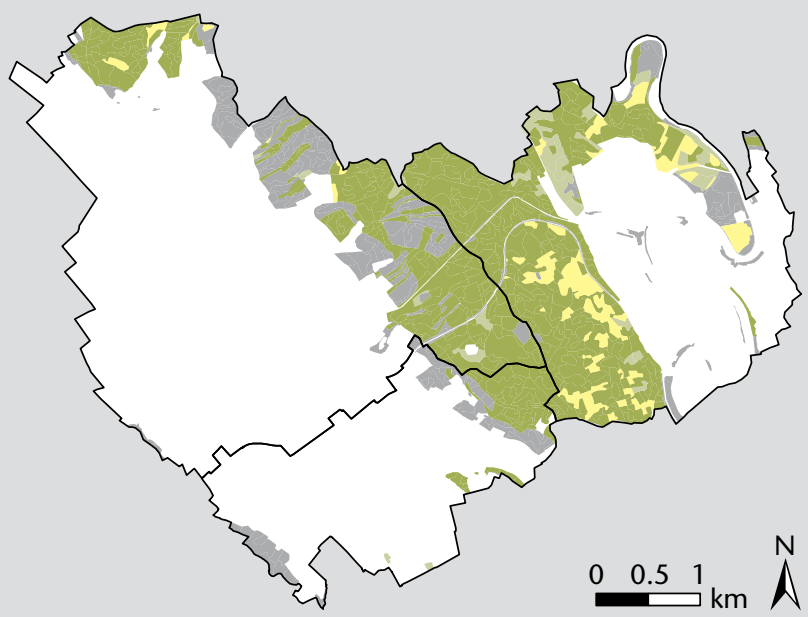

Variante Energieholz, Vollbaum Winter

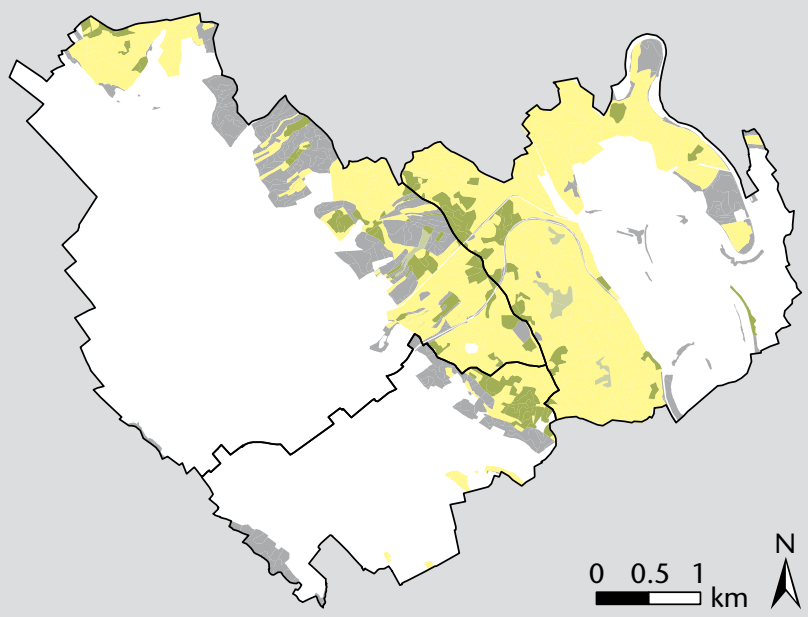

keine Angabe ungefährdet indifferent leicht gefährdet gefährdet

Abb 7 Bewertung der Standortgefährdung bei unterschiedlichen Waldbau- und Aushaltungsvarianten im Forstbetrieb Bremgarten, Wohlen, Waltenschwil (BWW). Die grauen Flächen (keine Angabe) betreffen hauptsächlich den Privatwald auf Territorium der politischen Gemeinden Bremgarten, Wohlen und Waltenschwil.

tes als unproblematisch und nur auf einer kleinen Fläche als indifferent anzusehen (Abbildung 8). Dagegen muss vielerorts mit Kaliummangel gerechnet werden. Auf einigen Flächen ist auch die Nachhaltigkeit in Bezug auf den NGV für Mangan nicht gegeben. Phosphor weist zwar keine problematische, aber viele indifferente Flächen auf, was auch zur Vorsicht mahnt. Zudem gibt es einige als unproblematisch eingestufte Flächen (hellgrün), auf denen der Phosphorvorrat über eine Zeitdauer von mehr als zehn Umtriebszeiten abgebaut wird, was definitionsgemäss als nachhaltig eingestuft wurde. Auf einem Grossteil des Forstbetriebes ist der Schwefel bei einer Vollbaumernte unproblematisch. Es ist jedoch zu bedenken, dass diese vier Nährstoffe zu den wichtigen bis mittelmässig wichtigen Nährstoffen für das Pflanzenwachstum gehören und für den Energiehaushalt, für Enzymreaktionen und als osmotisch wirksame Komponenten, für die Assimilation und für die Struktur sowie die Funktion der Zellwände benötigt werden (Kilian et al 1994).

\section{Fazit Fallbeispiel}

Zusammenfassend kann festgestellt werden, dass zwischen den einzelnen Aushaltungsvarianten beträchtliche Unterschiede bezüglich der Standortgefährdung bestehen. Ist die Derbholznutzung in den meisten Fällen bedenkenlos, so müssen bei einer Vollbaumnutzung die lokalen ökologischen Gegebenheiten berücksichtigt werden. Auf jeden Fall sollte auf den Flächen, die als «indifferent» bewertet wurden, vorsichtshalber keine Vollbaumnutzung durchgeführt werden, bis genauere Beurteilungsgrundlagen vorliegen.

Der Forstbetriebsleiter muss bei jedem Standort abwägen, ob die zusätzlichen Risiken der Vollbaumnutzung in Kauf genommen werden sollen, um die Nutzungsmengen zu erhöhen. Das hier vor- 


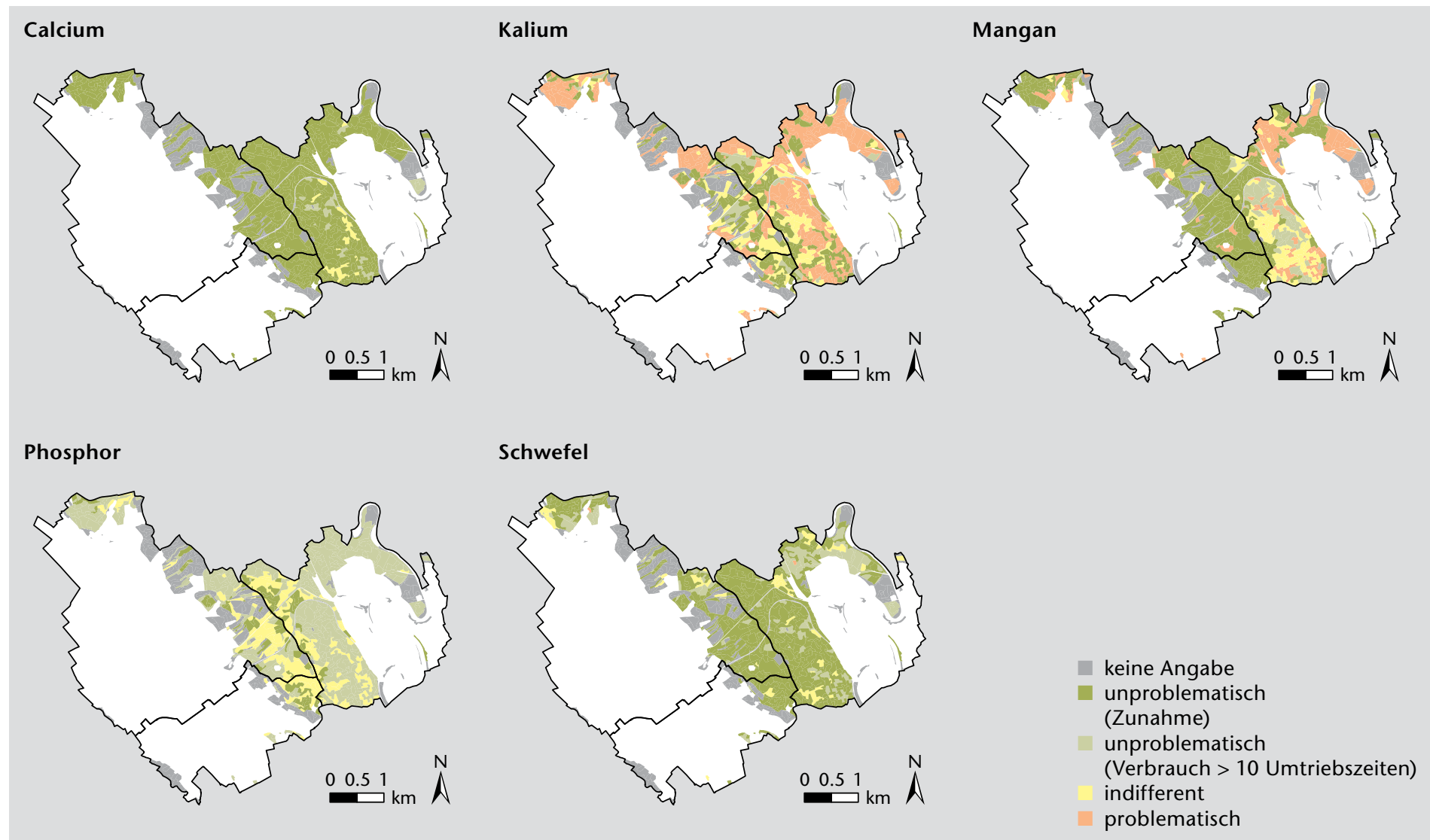

Abb 8 Ungewichtete Bewertung einzelner Nährstoffe bei der Waldbau- und Aushaltungsvariante Wertholz (Wh), Vollbaum Winter (VBW) im Forstbetrieb Bremgarten, Wohlen, Waltenschwil (BWW) mithilfe des Nährstoffbilanzierungsmodells (NBM). Die grauen Flächen (keine Angabe) betreffen hauptsächlich den Privatwald auf Territorium der politischen Gemeinden Bremgarten, Wohlen und Waltenschwil.

gestellte Nährstoffbilanzierungsmodell kann ihm dabei wertvolle Dienste leisten.

\section{Diskussion und Folgerungen}

Mit dem NBM steht ein GIS-gestütztes Tool zur Verfügung, um auf der Ebene des Bestandes Biomasse- und Nährstoffentzug im Rahmen der Holzernte bei unterschiedlichen Waldbau- und Aushaltungsvarianten zu ermitteln und die Standortgefährdung zu visualisieren. Die vorhandenen Grundlagen und Modellansätze reichen aus, um dazu erste Aussagen zu machen, weisen zurzeit jedoch noch verschiedene Schwachstellen auf, die es nach Möglichkeit zu beheben gilt:

Das NBM bedient sich verschiedener geprüfter Modelle, wie des Bestandesentwicklungsmodells des Forstbetriebssimulationsmodells (FBSM; Erni \& Lemm 1995), des Verwitterungsmodells von Sverdrup (1990), des Lufteintragsmodells für Stickstoff (Bafu 2007), sowie des Auswaschungs- und Ernteexportmodells (Hässig et al 2009). Dank der Integration des Bestandesentwicklungsmodells konnte die Anwendbarkeit des NBM deutlich erweitert werden. Waldbauliche und erntetechnische Varianten lassen sich dadurch einfach prognostizieren. Die Grenzen werden allerdings durch die vereinfachenden Annahmen, dass Nadelholz durch Fichte und
Laubholz durch Buche repräsentiert wird, und durch die Abbildungstreue des Waldwachstumssimulators vorgegeben.

Die Zerlegung des Baumes in einzelne Kompartimente und die Berücksichtigung des Ein- und Austrages und des Vorrates von Nährstoffen stellen einen wichtigen Schritt in der Bewertung der Standortgefährdung dar, welcher in bisherigen Studien meist übergangen wurde. Der Bilanzrahmen deckt jedoch nicht alle Nährstoffprozesse rund um den Wald genügend ab. Vor allem sind die Vorgänge im Boden nur grob erfasst. Auch die Nährstoffgehalte in den Baumkompartimenten variieren je nach Baumart, Jahrringbreite und Nährelement stark und können sogar standortspezifisch sehr unterschiedlich ausfallen (Jacobsen et al 2002). Hier braucht es weitere Forschungsarbeiten, wenn man die Vorhersagegenauigkeit bezüglich der Nährstoffentzüge verbessern will.

Zurzeit werden der Bodentyp und damit die Nährstoffvorräte im Boden über die Waldgesellschaft zugeordnet. Über diese Zuordnung werden auch Auswaschung und Verwitterung berechnet, womit drei wesentliche Bilanzgrössen von dieser $\mathrm{Zu}$ ordnung abhängen. Hier liegt die grösste Schwäche. Um die Zuverlässigkeit der Prognose zu erhöhen, müssten die Datenbasis der bodenkundlichen Kennwerte (Nährstoffvorräte, Verwitterungs- und Auswaschungsraten) verbessert und geeignete Methoden 
zur flächigen Extrapolation dieser Grössen gefunden werden. Dies kann durch eine Erweiterung der nationalen Bodendaten mit kantonalen Erhebungen geschehen, wodurch mehr Stützwerte für eine direkte flächige Extrapolation der relevanten Bodenkennwerte vorhanden wären. Zudem erlaubt dies, das Modell in Regionen mit besonders guter Datenbasis zu verfeinern.

Die im NBM verwendeten Lufteintragsdaten aus dem Schwarzwald für Mangan, Eisen und Phosphor vermögen die Verhältnisse in den Alpen nur ungenügend abzubilden. Im Rahmen der Weiterentwicklung des Modells ist zu prüfen, ob mit Level-IIDaten aus Deutschland und Österreich die Datenbasis verbessert werden könnte.

Die Nährstoffvorräte der Schweizer Waldböden sind relativ gut bekannt. Es gibt rund 1000 Bodenprofile, bei welchen die Vorräte erhoben respektive berechnet wurden. Die Extrapolation dieser Daten auf die Fläche sollte für zukünftige Anwendungen verbessert werden. Da die pflanzensoziologischen Einheiten bezüglich Bodenkennwerten sehr grosse Streuungen aufweisen, müssen für die flächige Extrapolation bodenkundlicher Daten bessere Hilfsgrössen gefunden werden. Auch die Depositionsraten stimmen recht gut, da sie relativ einfach zu messen sind. Will man die Deposition nicht nur für die Forstregion, sondern für kleinere Regionen haben, so ist ebenfalls das Messstellennetz zu verdichten.

Sowohl die Bilanzierungsergebnisse als auch ihre Bewertung sind im Hinblick auf die Relevanz für das Pflanzenwachstum erneut zu überprüfen. Dabei sind insbesondere offene Fragen bezüglich der Bioverfügbarkeit der Nährstoffe, im Speziellen beim Phosphor, zu klären.

Im Moment werden im Modell für die Aushaltungsvariante Vollbaum 1) alle waldbaulichen Eingriffe während einer Umtriebszeit als Vollbaumnutzungen geführt und 2) alle Bäume vollständig als Vollbäume (abzüglich Ernteverlust) entnommen. In Realität wird man die Vollbaumnutzung aus ökologischen und ökonomischen Gründen differenziert gestalten, d.h., nicht bei jedem Eingriff wird man Vollbäume nutzen oder Äste, Reisig und Blätter eines Baumes vollumfänglich entnehmen. Letzteres könnte im Modell auf einfache Art berücksichtigt werden.

Für das Systemverständnis und eine differenzierte Gestaltung von Waldbau und Erntetechnik auf quantitativer Basis bildet das NBM ein wertvolles Instrument. Es erhält durch die aktuelle Diskussion der Rückführung von Holzasche in den Wald zusätzliche Bedeutung. Der Aussagekraft des Modells und seiner Praxistauglichkeit sind zurzeit jedoch durch die oben diskutierten Einschränkungen enge Grenzen gesetzt. Sie können durch verbesserte Grundlagen und verfeinerte Modellansätze verbessert werden.

Eingereicht: 9. April 2010, akzeptiert (mit Review): 29. Juni 2010

\section{Literatur}

BAFU (2007) Stickstoff-Deposition 2000. Bern: Bundesamt Umwelt. stratus.meteotest.ch/lugis/map/presentation/lugis_style/map.asp?Cmd=zoom In\&MapURL=\&MinX $=475$ $723637 \& \operatorname{Min} Y=84892808 \& \operatorname{Max} X=845646362 \& \operatorname{Max} Y=$ $275221191 \&$ lang $=d \& S$ chadstoff $=$ NDEP_00\&id $x=29 \&$ Zoomfaktor=1 (5.7.2010).

BFS (2002) Umwelt Schweiz 2002: Statistiken und Analysen. Neuenburg: Bundesamt Statistik. 322 p.

BLASER P, ZIMMERMANN S, LUSTER J, WALTHERT L, LÜSCHER P (2005) Waldböden der Schweiz. Band 2. Regionen Alpen und Alpensüdseite. Bern: Hep. 920 p.

BRÄNDLI UB ET AL (2001) Swiss national forest inventory: Methods and models of the second assessment. Birmensdorf: Eidgenöss Forsch.anstalt Wald Schnee Landsch. $312 \mathrm{p}$.

BRÄNDLI UB, EDITOR (2010) Schweizerisches Landesforstinventar. Ergebnisse der dritten Erhebung 2004-2006. Birmensdorf: Eidgenöss Forsch.anstalt Wald Schnee Landsch. $312 \mathrm{p}$.

ELLENBERG H, KLÖTZLI F (1972) Waldgesellschaften und Waldstandorte in der Schweiz. Mitt Schweiz Anst Forstl Versuchsw 48: 587-930.

ERNI V, FRUTIG F (2004) «HeProMo» - für die einfache Vorkalkulation von Holzerntearbeiten. Wald Holz 85 (5): 46-47.

ERNI V, LEMM R (1995) Ein Simulationsmodell für den Forstbetrieb - Entwurf, Realisierung und Anwendung. Birmensdorf: Eidgenöss Forsch.anstalt Wald Schnee Landsch, Bericht $341.89 \mathrm{p}$.

GÖTTLEIN A, ETTL R, WEIS W (2007) Energieholznutzung und nachhaltige Waldbewirtschaftung - ein Zielkonflikt? München: Bayer Akademie Wissenschaften, Rundgespräche Kommission Ökologie 33. pp. 87-95.

HÄSSIG J, LEMM R, THEES O, WALDNER P, ZIMMERMANN S (2009) Bewertung der Nachhaltigkeit des Nährstoffentzugs durch die Holzernte - Erste Modellanwendung bei intensivierter Energieholznutzung im Schweizer Mittelland. In: Thees $\mathrm{O}$, Lemm R, editors. Management zukunftsfähige Waldnutzung. Birmensdorf: Eidgenöss Forsch.anstalt Wald Schnee Landsch. pp. 191-224.

HEYN B (1989) Elementflüsse und Elementbilanzen in Waldökosystemen der Bärhalde - Südschwarzwald. Freiburg i.Br.: Institut Bodenkunde Waldernährungslehre, Freibg bodenkundl Abh 23. 189 p.

JACOBSEN C, RADEMACHER P, MEESENBURG H, MEIWES KJ (2002) Gehalte chemischer Elemente in Baumkompartimenten. Literaturstudie und Datensammlung. Göttingen: Niedersächsische Forstliche Versuchsanstalt. $81 \mathrm{p}$.

KILIAN W ET AL (1994) Die Düngung im Wald, 2. Teil. Anleitung zur Walddüngung. Wien: Bundesministerium LandForstwirtschaft, Fachbeirat Bodenfruchtbarkeit Bodenschutz. 41 p.

LEMM R, ERNI V (2009) Bessere Nutzungs- und Allokationsentscheide mit dem Sortimentssimulator SORSIM. In: Thees $\mathrm{O}$, Lemm R, editors. Management zukunftsfähige Waldnutzung. Birmensdorf: Eidgenöss Forsch.anstalt Wald Schnee Landsch. pp. 415-440.

LEMM R, ERNI V, THEES O (2002) Komponentenbasierte Softwareentwicklung - neue Perspektiven für forstliche Modellierung und Informationsverarbeitung. Schweiz Z Forstwes 15: 3-9. doi: 10.3188/szf.2002.0003

LEMM R, ERNI V, FRUTIG F, OSWALD K, THEES O (2000) Produktivitätsmodelle für Verjüngung, Jungwaldpflege und Holzernte mit Hilfe komponentenbasierter Softwaretechnologie. Schlussbericht zum Projekt Nr. 98.03 des Wald- und 
Holzforschungsförderungsfonds. Birmensdorf: Eidgenöss Forsch.anstalt Wald Schnee Landsch. 71 p.

MAZZARINO MJ (1981) Holozäne Silikatverwitterung in mitteldeutschen Waldböden aus Löss. Göttingen: Univ Göttingen, Landwirtschaftliche Fakultät, Dissertation. 188 p.

RADEMACHER P, MÜLLER-USING B (2004) Bioelemententzug bei der Holznutzung in Rein- und Mittelbeständen aus Buche und Fichte. Teilvorhabenbericht. In: Indikatoren und Strategien für eine nachhaltige, multifunktionale Waldnutzung - Fallstudie Waldlandschaft Solling. Göttingen: Univ Göttingen, Forschungszentrum Waldökosysteme, Ber B 71. pp. 81-110.

RIHM B (1994) Critical loads of acidity for forest soils and alpine lakes. Steady state mass balance method. Bern: Federal Office of Environment, Forests and Landscape, Environmental Series 234. 68 p.

ROSSET C ET AL (2009) Potenziale, Verfügbarkeit und Allokation des Schweizer Holzes - Aufbau und Anwendung der Dynamischen Waldholzverfügbarkeitskarte WVK. In: Thees $\mathrm{O}$, Lemm R, editors. Management zukunftsfähige Waldnutzung. Birmensdorf: Eidgenöss Forsch.anstalt Wald Schnee Landsch. pp. 157-190.

SCHMIDT O (2003) Der Energieinhalt von Holz und seine Bewertung. Freising: Bayer Landesanstalt Wald Forstwirtschaft (LWF), Merkblatt 12. 4 p.

\section{Ein Modell zur Bilanzierung des holzerntebedingten Nährstoffentzugs auf Schweizer Waldböden}

Um die Risiken des Nährstoffentzugs durch Holzernte auf quantitativer Basis zu beurteilen, wurde ein Nährstoffbilanzierungsmodell (NBM) für die Schweiz entwickelt. Es lässt sich innerhalb von Forstbetrieben und grösseren Regionen anwenden. Das Modell bilanziert den Nährstoffentzug auf der Ebene des einzelnen Waldbestandes im Rahmen einer Szenarioanalyse. Dabei wird der erntebedingte Entzug von neun Nährstoffen unter verschiedenen Waldbau- und Holzaushaltungsvarianten quantifiziert und in Beziehung zu den Potenzialen im Boden und deren Veränderungen gesetzt. Die Ergebnisse der Simulationen werden einer Bewertung unterworfen, welche sich an zwei Nachhaltigkeitskriterien orientiert und zusammenfassend die Gefährdung des Standortes bezüglich des Nährstoffmangels beschreibt. Wegen fehlender Grundlagen sind der Aussagekraft des Modells bei der praktischen Anwendung derzeit allerdings noch Grenzen gesetzt. Der Beitrag zeigt den Aufbau und die Funktionsweise des Modells, skizziert die IT-Umsetzung, visualisiert die Ergebnisse der ersten Anwendung im Forstbetrieb Bremgarten, Wohlen, Waltenschwil und mündet in eine kritische Analyse der bisherigen Erfahrungen.
SEIFERT T, SCHUCK J, BLOCK J, PRETZSCH H (2006) Simulation von Biomasse- und Nährstoffgehalt von Waldbäumen. In: Nagel J, editor. Beiträge zur Jahrestagung vom 29.-31. Mai 2006. Göttingen: Deutscher Verband Forstlicher Forschungsanstalten, Sektion Ertragskunde. pp. 208-223.

SIGG L, STUMM W (1994) Aquatische Chemie: eine Einführung in die Chemie wässriger Lösungen und natürlicher Gewässer. Zürich: VDF, 3 ed. 498 p.

SVERDRUP HU (1990) The kinetics of base cation release due to the chemical weathering. Lund: Departement of Chemical Engineering II, Lund Institute of Technology. 246 p.

THIMONIER A, SCHMITT M, WALDNER P, RIHM B (2005) Atmospheric deposition on Swiss long-term forest ecosystem research (LWF) plots. Environ Monit Assess 104: 81-118.

WALTHERT L, ZIMMERMANN S, BLASER P, LUSTER J, LÜSCHER P (2004) Waldböden der Schweiz. Band 1. Grundlagen und Region Jura. Bern: Hep. 768 p.

WITTKOPF S (2005) Bereitstellung von Hackgut zur thermischen Verwertung durch Forstbetriebe. Freising: Fakultät Wissenschaftszentrum Weihenstephan für Ernährung, Landnutzung und Umwelt. $189 \mathrm{p}$.

ZIMMERMANN S, LUSTER J, BLASER P, WALTHERT L, LÜSCHER P (2006) Waldböden der Schweiz. Band 3. Regionen Mittelland und Voralpen. Bern: Hep. 848 p.

\section{Un modèle pour établir le bilan des prélèvements d'éléments nutritifs dans les sols forestiers suisses en rapport avec la récolte du bois}

Un modèle (NBM) destiné à établir un bilan quantitatif des éléments nutritifs dans le sol a été développé pour la Suisse afin de connaître le risque lié aux prélèvements de bois. Ce modèle s'applique à l'intérieur des entreprises forestières ainsi que des grandes régions, et permet, grâce à une analyse de scénarios, d'établir le bilan du prélèvement d'éléments nutritifs à l'échelle du peuplement. Ainsi quantifie-t-on le prélèvement de neuf éléments, en considérant diverses variantes de sylviculture et de préparation du bois, et en établissant le rapport avec les potentialités du sol et de son évolution. Les résultats de cette simulation sont ensuite mis en rapport avec deux critères de durabilité afin de décrire globalement la dangerosité du prélèvement d'éléments nutritifs. Mais pour l'instant, la pertinence des résultats obtenus est encore limitée lors des applications pratiques, en raison du manque de données de base. L'article décrit la structure et le fonctionnement du modèle, esquisse l'application numérique, présente les résultats de la première application dans les exploitations forestières de Bremgarten, Wohlen et Waltenschwil, et s'achève par une analyse critique des expériences réalisées jusqu'ici. 